\title{
Nonlinear generation of vector beams from AlGaAs nanoantennas
}

\author{
Rocio Camacho, ${ }^{\dagger}$ Mohsen Rahmani, ${ }^{\dagger}$ Sergey Kruk, ${ }^{\dagger}$ Lei Wang, ${ }^{\dagger}$ Lei Xu ${ }^{\dagger, \dagger}$ Daria \\ Smirnova, ${ }^{\dagger}$ Alexander Solntsev, ${ }^{\dagger}$ Andrey Miroshnichenko, ${ }^{\dagger}$ Hoe Tan, ${ }^{\top}$ Fouad \\ Kaurota, "Shagufta Naureen, "Kaushal Vora, "Luca Carletti, ${ }^{\S}$ Costantino De \\ Angelis, ${ }^{\S}$ Chennupati Jagadish, $₫$ Yuri S. Kivshar, ${ }^{\dagger}$ and Dragomir N. Neshev*,† \\ $\dagger$ Nonlinear Physics Centre, Research School of Physics and Engineering, The Australian \\ National University, Canberra ACT 2601, Australia \\ $\ddagger$ The MOE Key Laboratory of Weak Light Nonlinear Photonics, School of Physics and \\ TEDA Applied Physics Institute, Nankai University, Tianjin 300457, China \\ \Department of Electronic Materials Engineering, Research School of Physics and \\ Engineering, The Australian National University, Canberra ACT 2601, Australia \\ $\S$ Department of Information Engineering, University of Brescia, Via Branze 38, 25123 \\ Brescia, Italy \\ E-mail: Dragomir.Neshev@anu.edu.au
}

\begin{abstract}
The quest for nanoscale light sources with designer radiation patterns and polarization has motivated the development of nanoantennas that interact strongly with the incoming light and are able to transform its frequency, radiation and polarization patterns. Here, we demonstrate dielectric AlGaAs nanoantennas for efficient second harmonic generation, enabling the control of both directionality and polarization of
\end{abstract}


nonlinear emission. We show that the nanodisk AlGaAs antennas can emit second harmonic in preferential directions and generate complex vector polarization beams, including beams with radial polarization. This is enabled by specialized III-V semiconductor nanofabrication of quality AlGaAs nanostructures embedded in low-index material, thus allowing for simultaneous forward and backward nonlinear emission. Our results represent a fundamental step towards efficient nonlinear nanoscale light sources, finding important applications in bio-photonic imaging and as elements for efficient nonlinear holograms.

KEYWORDS: nonlinear optics, second harmonic generation, dielectric nanoantennas, nanophotonics, III-V semiconductors, nanofabrication

In recent years, the use of nanoparticles for photon management at the nanoscale has attracted enormous interest in the scientific community. Enabling new optical functionalities at the nanoscale requires nanophotonic components capable of manipulating light locally and then of re-emitting the energy with on-demand frequency, radiation and polarization patterns. ${ }^{1}$ We have thus witnessed the development of a plethora of nanoscale metallic and/or dielectric photonic components, operating as optical nanoantennas for different applications, including control of light scattering, ${ }^{2-4}$ and control of quantum emission, ${ }^{5}$ such as radiation pattern $^{6}$ and polarization. ${ }^{7}$

However, when active control over the radiation frequency is required, new challenges are to be faced, as this is locked to the emitter's transitions and cannot be changed by the excitation. While research is underway to overcome this limitation by realising strong coupling between the emitter and the antenna, ${ }^{8}$ a lot more flexibility in the frequency conversion can be realised through nonlinear interactions between the light and the nanoantennas, including nonlinear wave-mixing ${ }^{9-11}$ and harmonic generation. ${ }^{12,13}$ Indeed, shaping of the nonlinear fields in the harmonic generation process by plasmonic nanoantennas has been recently tested. ${ }^{14,15}$ However, the observed efficiency of the nonlinear frequency conversion remains small, of the order of $\sim 10^{-11}$, despite the implementation of different strategies to boost it, 
such as resonant coupling, ${ }^{16-20}$ three-dimensional geometries, ${ }^{13}$ or hybrid nanoantennas. ${ }^{21}$

All-dielectric nanoantennas ${ }^{22}$ have recently been suggested as an important pathway to enhance the frequency conversion efficiency beyond what is possible with plasmonics. ${ }^{23,24}$ The negligible resistive losses of dielectric nanoantennas avoid heating problems and allow excitation at much higher light intensities, which is of paramount importance for the efficiency of nonlinear optical phenomena. ${ }^{25}$ Indeed, third-harmonic generation (THG) in Si and Ge antennas has been recently investigated, showing a huge enhancement of the conversion efficiency by optically pumping near the antenna's magnetic dipolar ${ }^{23,26}$ or anapole resonances. ${ }^{27}$ Conversion efficiencies of $10^{-7}$ (see Ref. ${ }^{23}$ ) and $10^{-6}$ (see Ref. ${ }^{27,28}$ ) have been achieved experimentally. These results clearly illustrate the potential of all-dielectric nanoparticles for nonlinear nanophotonic applications.

Despite these spectacular achievements, only $\chi^{(3)}$ nonlinear effects have been observed in these platforms, since Si and Ge do not exhibit bulk quadratic optical nonlinearity, because of their centro-symmetric crystal structure. However, exploiting materials with second-order nonlinear susceptibilities, such as GaAs and AlGaAs, would intrinsically increase the conversion efficiency due to the lower-order nonlinearity. The nonlinear effects in GaAs nanostructures have been the subject of continuing research, including SHG in GaAs nanowires, ${ }^{29-31}$ hybrid GaAs-plasmonic nanoholes, ${ }^{32}$ GaAs micro-ring resonators on insulator ${ }^{33}$ and most recently dielectric nanoantennas and metasurfaces. ${ }^{24,34-36}$ SHG efficiencies larger than $10^{-3}$ have been theoretically predicted for free standing AlGaAs nanoantennas ${ }^{24}$ and efficiencies exceeding $\sim 10^{-5}$ have been recently measured in backward scattering for $\mathrm{AlGaAs}^{35}$ and $\mathrm{GaAs}^{36}$ sitting on an oxide layer. However, no experiments to date have tested the possibility for shaping the radiation and polarization patterns of $S H G$, including achieving unidirectional harmonic generation or nonlinear generation of beams of complex polarization.

Here, we obtain efficient second harmonic generation (SHG) from dielectric AlGaAs nanoantenna, and demonstrate the possibility of shaping the SH radiation pattern in forward and backward directions, as well as its polarization state. In particular we prove that the 
SHG emission has a complex spatial distribution of its polarization state, which for a specific size of the nanoantennas leads to the generation of cylindrical vector beams of radial polarization. ${ }^{37}$ The properties of our SHG aerials are enabled thanks to a new AlGaAs platform, which allows for simultaneous forward and backward radiation. Our results demonstrate for the first time to our knowledge the functional beam and polarization shaping of the nonlinear second harmonic emission from dielectric nanoantennas. This represents a fundamental step for the manipulation of the angular emission of AlGaAs nanoantennas, which will foster future studies toward the goal of precision engineering of their nonlinear radiation pattern.

An important strategy to achieve such full control of harmonic radiation is to be able to fabricate AlGaAs nanoantennas that are accessible from forward and backward directions. However, the existing fabrication techniques do not allow for this, as they require a nontransparent III-V handle wafer for direct growth of III-V semiconductors. The growth on transparent substrates (e.g. glass) is avoided ${ }^{33,35,36}$ because it results in a high density of dislocations. Recent works have been devoted to transfer of semiconductor films onto a glass substrate, followed by the fabrication of micro/nano structures, ${ }^{3,33}$ however this method does not allow for fabrication of high resolution nanostructures with smooth surfaces and edges, which is crucial for the exploration of the bulk SHG from AlGaAs nanoantennas, as our goal.

Here we implemented a novel fabrication procedure of AlGaAs-in-insulator, containing epitaxial growing technique in conjunction with a bonding procedure to a glass substrate. Our final sample contains high quality $\mathrm{Al}_{0.2} \mathrm{Ga}_{0.8}$ As nanodisks partially embedded in transparent Benzocyclobutene (BCB) layer, with equivalent refractive index to glass, on a glass substrate. Figures 1(a-d) illustrate the fabrication steps (details can be found in Methods). Electron microscopy images of AlGaAs disks embedded in the BCB and the main sample can be seen in Figs. 1(e) and 1(f), respectively. Further images on the fabrication steps can be found in the Supporting Information.

The AlGaAs nanodisks are designed to support Mie-type resonances at both the fundamental wave $(\mathrm{FW})$ and the second harmonic $(\mathrm{SH})$. In order to match the excitation of the 


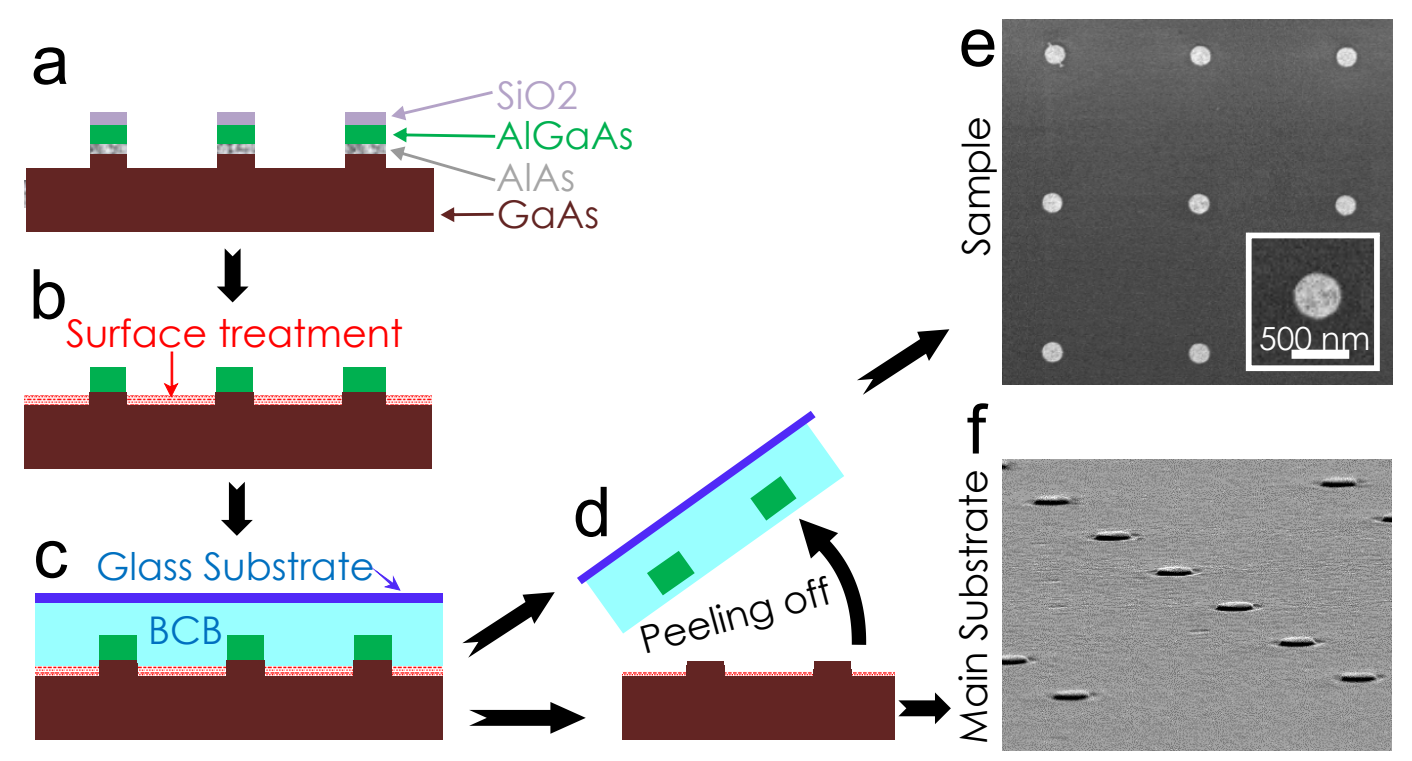

Figure 1: Fabrication procedure for AlGaAs nanoresonators in a transparent media. (a) AlGaAs nanodisks defined on a GaAs wafer via electron beam lithography and sequential etching. $\mathrm{SiO}_{2}$ is used as a mask and AlAs as a buffer layer. (b) Formation of a non-adhesive surface with $\mathrm{Cl}_{2}$ treatment. Sequential removal of the $\mathrm{SiO}_{2}$ mask and $\mathrm{AlAs}$ buffer layer by $\mathrm{HF}$ acid. (c) Coating of a $\mathrm{BCB}$ layer followed by curing and bonding it to a thin glass substrate. (d) Peeling off the AlGaAs nanoresonators embedded into BCB. SEM images: (e) top-view of the final sample and (f) side view of the main GaAs wafer.

Mie-resonant modes to the FW and SH frequencies, we fabricate nanodisks of various diameters in the range $340-690 \mathrm{~nm}$ at a fixed height of $300 \mathrm{~nm}$. Two sets of samples are fabricated: (i) arrays of nanodisk antennas $(1 \mu \mathrm{m}$ periodicity) for linear optical characterization; and (ii) single nanodisks ( $5 \mu \mathrm{m}$ apart) for SHG experiments.

First, we measure the linear transmission spectra of the different arrays, Fig. 2(a) (see details in Methods). The measured spectra are shown in Fig. 2(b). The two vertical dashed lines indicate the FW and SH wavelengths. We observe a pronounced size-dependant resonance at the FW wavelength and multiple resonances at the $\mathrm{SH}$ wavelength. This is confirmed by numerical simulations using the rigorous coupled wave analysis (RCWA) method. ${ }^{38}$ The calculated zero-order forward scattering spectra are depicted in Fig. 2(c). The experimentally measured spectra are in a good agreement with our numerical calculations.

In Fig. 2(d) we show the extracted scattering cross-section at the FW for nanodisks of different diameters (see details in Methods). Dots indicate the experimental measurements 

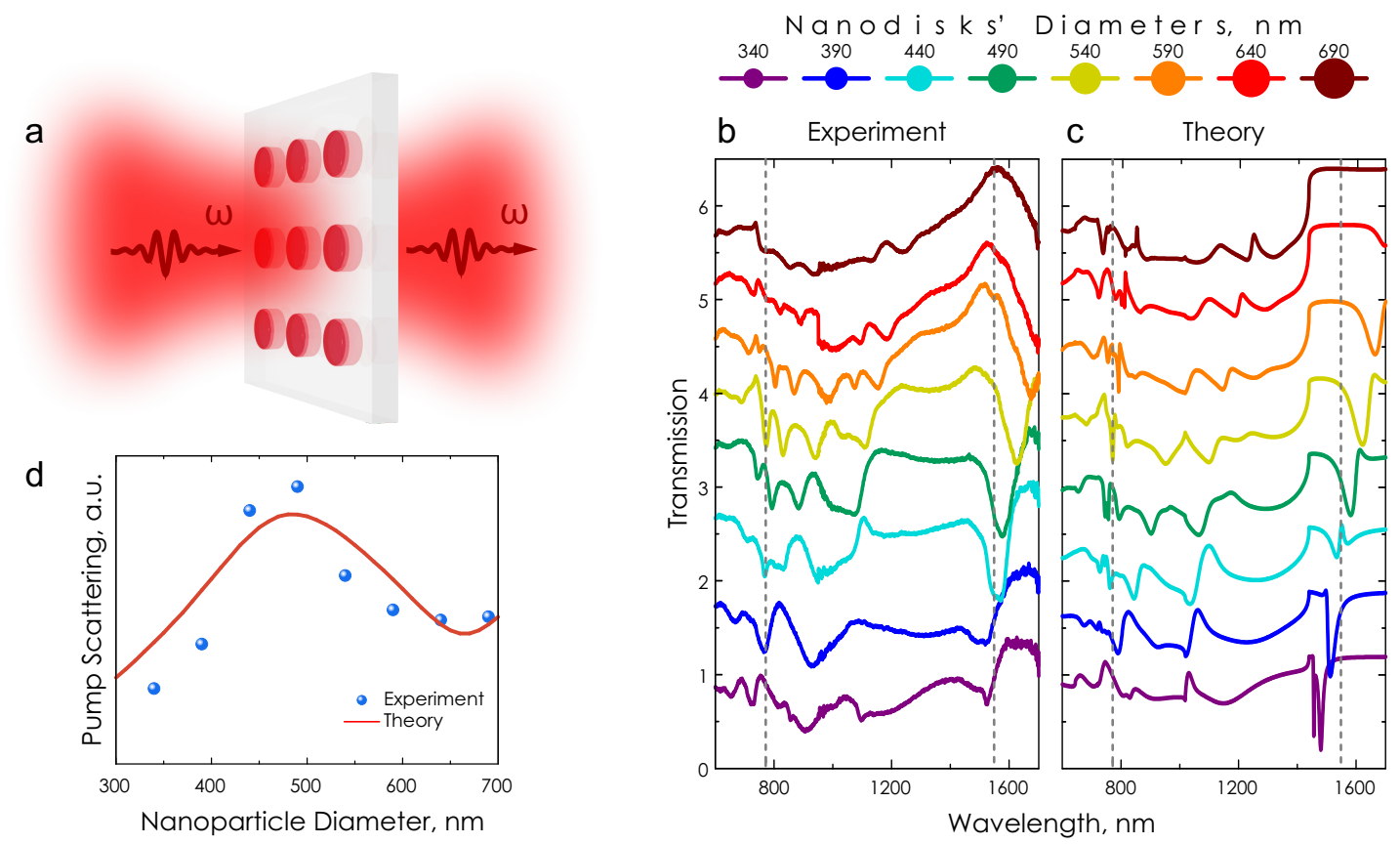

Figure 2: Linear spectroscopy of AlGaAs resonant nanoantennas. (a) Schematic of transmission measurements of an array of nanodisk antennas. (b and c) Transmission spectra of the nanodisk arrays measured experimentally and calculated theoretically, respectively. Different colors correspond to different diameters of the nanodisks. Dashed lines show the spectral positions of the FW and the SH. (d) Linear scattering of nanodisks of different diameters at pump wavelength of $1556 \mathrm{~nm}$. Solid line - theoretical calculations, dots experimental measurements.

and solid curve the numerical simulations. The resonant profile of the linear scattering, maximized for disk diameters of $400-500 \mathrm{~nm}$, is essentially determined by a magnetic dipole and a weaker electric dipole excitations in the disk, playing a dominant role at the pump FW wavelength $(1556 \mathrm{~nm})$. Some minor contributions of quadrupoles tend to grow slightly, when increasing the disk radius. At the $\mathrm{SH}$ wavelength $(778 \mathrm{~nm})$, higher-order multipoles are excited in the nanodisks. These two resonant conditions at the FW and the SH wavelength are responsible for SHG enhancement in our nanoantennas. However, a more sophisticated dependence of the SHG efficiency on the size of the nanodisk is expected when taking into account the spatial overlaps of the resonant modes at the FW and the SH fields.

While these results are obtained for arrays of nanodisks, the relatively large period of $1 \mu \mathrm{m}$ assures little influence on the nearest neighbour coupling. Although, some red shift in 
the maximal scattering efficiency can be expected for isolated nanodisks. Therefore, from these results we can infer the optimal sizes of individual AlGaAs nanodisk to explore for achieving maximum efficiency of SHG from single nanoantennas.

Next, we use AlGaAs nanoantennas fabricated $5 \mu \mathrm{m}$ apart to be able to detect their individual nonlinear response, Fig. 3(a). We measure the efficiencies of the SHG in both forward and backward directions for various sizes of the nanodisks (see details in Methods). These measurements are only possible due to the transparent and homogeneous surrounding environment of the AlGaAs nanoantennas enabled by our fabrication. In the experiments we use linear vertical polarization of pump, which is at $45^{\circ}$ to the crystalline axes of the AlGaAs nanodisks in order to maximize the nonlinear tensor component. ${ }^{24,34}$ The laser beam of average beam power of $\sim 1 \mathrm{~mW}$ is then focused by an infrared objective $(\mathrm{NA}=0.85)$ to a diffraction limited spot of $2.2 \mu \mathrm{m}$, resulting in peak intensity of $\sim 7 \mathrm{GW} / \mathrm{cm}^{2}$. Another visible objective $(\mathrm{NA}=0.9)$ collects the $\mathrm{SH}$ from an individual disk in forward direction, while the focusing objective collects the SH radiation in backward direction. The SH signal is detected by two cooled CCD cameras, calibrated with a power meter. Further details on the measurement procedure are given in the Methods section and the experimental setup is depicted in the Supporting Information. The results of our SHG measurements form a single AlGaAs nanoantenna of different disk diameters are shown in Fig. 3(b,c). The measured SH efficiency is shown in Fig. 3(b) and is derived as the sum of the measured forward and backward SH signals. The overall dependence of the efficiency on the antenna size is complex due to the large number of higher-order modes existing in the SH frequency band. The most efficient SHG is observed for the antenna diameter of $490 \mathrm{~nm}$, having conversion efficiency as high as $0.85 \times 10^{-4}$.

Importantly, as seen in Fig. 3(c), the directionality of the second harmonic emission can be tailored flexibly by changing the disk size. For example, for disk diameters about $400 \mathrm{~nm}$, the SH radiation is mostly backward, while for disk diameters $500-600 \mathrm{~nm}$, the backward to forward ratio remains close to unity with slight domination of the measured backward SH 

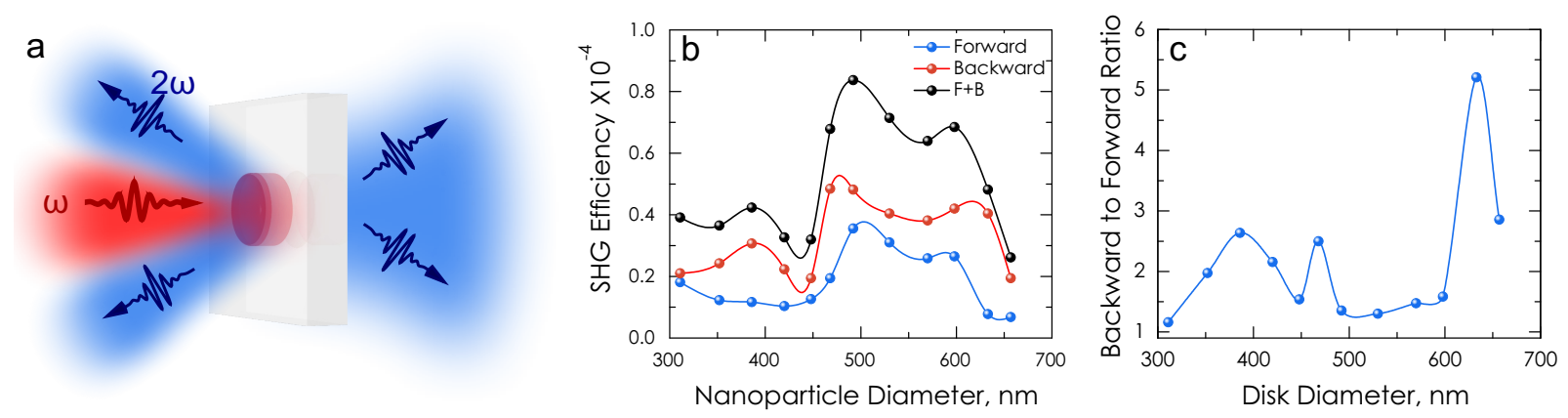

Figure 3: Nonlinear spectroscopy of single nanoantennas. (a) Schematic of the single antenna experiment. (b) Experimentally measured SHG efficiency $\left(P_{\mathrm{SH}} / P_{\mathrm{FW}}\right)$ from a single nanodisk of different diameter at pump wavelength of $1556 \mathrm{~nm}$. Blue - forward radiation, red - backward radiation. (c) Backward-to-Forward ratio of the SH.

emission. Note that at larger disk diameters, the backward-to-forward ration peaks again, however, for these larger disks, the dependence becomes very sensitive to the disk diameter due to the many higher order multipoles that contribute to the SH scattering. The key feature of our measurements is that we can characterize the nature of the radiation patterns in both directions, forwards and backwards, as well as in the transverse momentum, as recently predicted theoretically in Ref. ${ }^{34}$ Our data, however, suggest that the experimental measurement apparatus is capturing only a small portion of the total SH radiated power, due to the finite numerical aperture of our objectives.

To be able to estimate the total efficiency of the radiated SH power, we model numerically the nonlinear response of the AlGaAs nanodisks with the use of finite element method solver of COMSOL Multiphysics in the frequency domain. In our simulations, the disk is assumed to be embedded into a homogeneous medium with a refractive index equivalent to glass substrate. The material dispersion of AlGaAs is taken from the COMSOL tabulated data. The second-order nonlinear susceptibility tensor of the [001] grown AlGaAs, possessing a zinc blende crystalline structure, contains only off-diagonal elements $\chi_{i j k}^{(2)}$ with $i \neq j \neq k$. Thus, in the principal-axis system of the crystal, the $i$ th component of the nonlinear polarization at $\mathrm{SH}$ frequency is given by

$$
P_{i}^{(2 \omega)}=\varepsilon_{0} \chi_{i j k}^{(2)} E_{j}^{(\omega)} E_{k}^{(\omega)}
$$


We assume an undepleted pump approximation and follow the two coupled steps ${ }^{24,26,34,35}$ to calculate the radiated SH power. First, we simulate the linear scattering at the fundamental wavelength. To emulate the experimental conditions more accurately, the disk is excited by a focused monochromatic Gaussian beam, polarized along the [110] direction. The bulk nonlinear polarization (1) induced inside the particle is then employed as a source for the next electromagnetic simulation at the doubled frequency, to obtain the generated SH field.

We choose the disk size for maximal SH $d=490 \mathrm{~nm}$ and calculate the three-dimensional SH far-field radiation pattern, as shown in Fig. 4(a). The nonlinear scattering is governed by the interference of an electric quadrupole and higher-order nonlinearly-generated multipoles (up to $l=4$ ), leading to the suppression of the forward SH radiation, Fig. 4(a). The side, top and bottom views of this radiation pattern are also show in Figs. 4(b-d), respectively. The shaded area in Fig. 4(b) depicts the forward and backward collection angles of the SH signal in our experiments. These collection angles are also indicated by the inner circles in the forward and backward far-field radiation images in Figs. 4(c,d). Clearly, the collected energy in the experiment is less than the generated total SH. By integrating the amount of the SH emitted within the numerical aperture of the objective lenses used in experiment, we can determine that only about $30 \%$ of the total SH energy can be experimentally collected in forward and backward directions. As such, the total generation efficiency is estimated to be three times larger than the measured collection efficiency, thus exceeding the record value of $10^{-4}$. This high efficiency provides a solid ground for the use of our nonlinear nanoantennas as functional elements for nonlinear beam and polarization shaping.

Next, we perform experimental study of the radiation patterns. We build back-focal plane (BFP) images of the $\mathrm{SH}$ radiation pattern by adding a pair of confocal lenses between the objective lenses and the cameras, in both forward and backward directions. In Figs. 5(a,c - top row) we visualize portions of the radiation diagram captured by the objective lenses based on their numerical aperture. From these BFP images we can make the important conclusion that the $\mathrm{SH}$ radiation at normal direction (the $(0,0)$ point of the BFP images) 

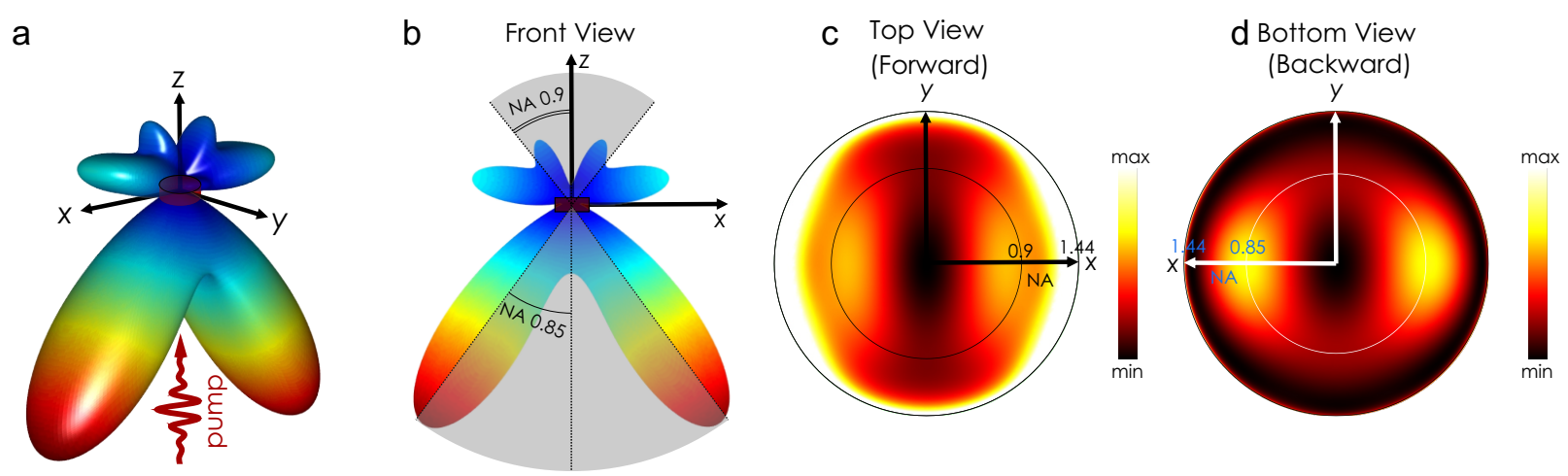

Figure 4: (a) Calculated 3D pattern of the Far-field SH radiation. (b) Front view of the pattern. Cones indicate range of angles experimentally accessible with our high-NA objectives. (c,d) Top and Bottom view of the radiation pattern (directionality diagrams) of the $\mathrm{SH}$ radiation within the experimentally accessible range of angles.

is zero, as recently predicted theoretically. ${ }^{34}$ Zero $\mathrm{SH}$ emission in normal direction here originates from the symmetry of the nonlinear bulk $\chi^{(2)}$ tensor and, thus, is not sensitive to geometry. As a result, the zero SH emission is observed for all studied AlGaAs nanodisks. To further support these findings, we also measure THG from the same disks. The third harmonic relies on $\chi^{(3)}$ nonlinear tensor and in contrast to the $\mathrm{SH}$ radiation pattern has radiation maximum in normal directions (see details in the Supporting Information). This is an important finding when arrays of such SH antennas are considered, as the interference of the multiple antennas from the array will result in lower efficiency radiation into the zero-th order SH beam. We also note that surface second-order nonlinearities can in principle result in normal $\mathrm{SH}$ radiation, ${ }^{36}$ however these are not pronounced in our experiments and the bulk $\chi^{(2)}$ is the dominant nonlinear contribution.

However, what is even more intriguing is the polarization state of the observed far-field doughnut beam. While most works to date have been focused on the radiation pattern of the emission, the polarization distribution of the emitted light emitted has never been studied before. To test the polarization properties of the $\mathrm{SH}$ radiation from our nanoantennas, we perform experimental retrieval of the spatially-resolved polarization states of the BFP images by using Stokes formalism (see details in Methods). We observe vector-beam formation 

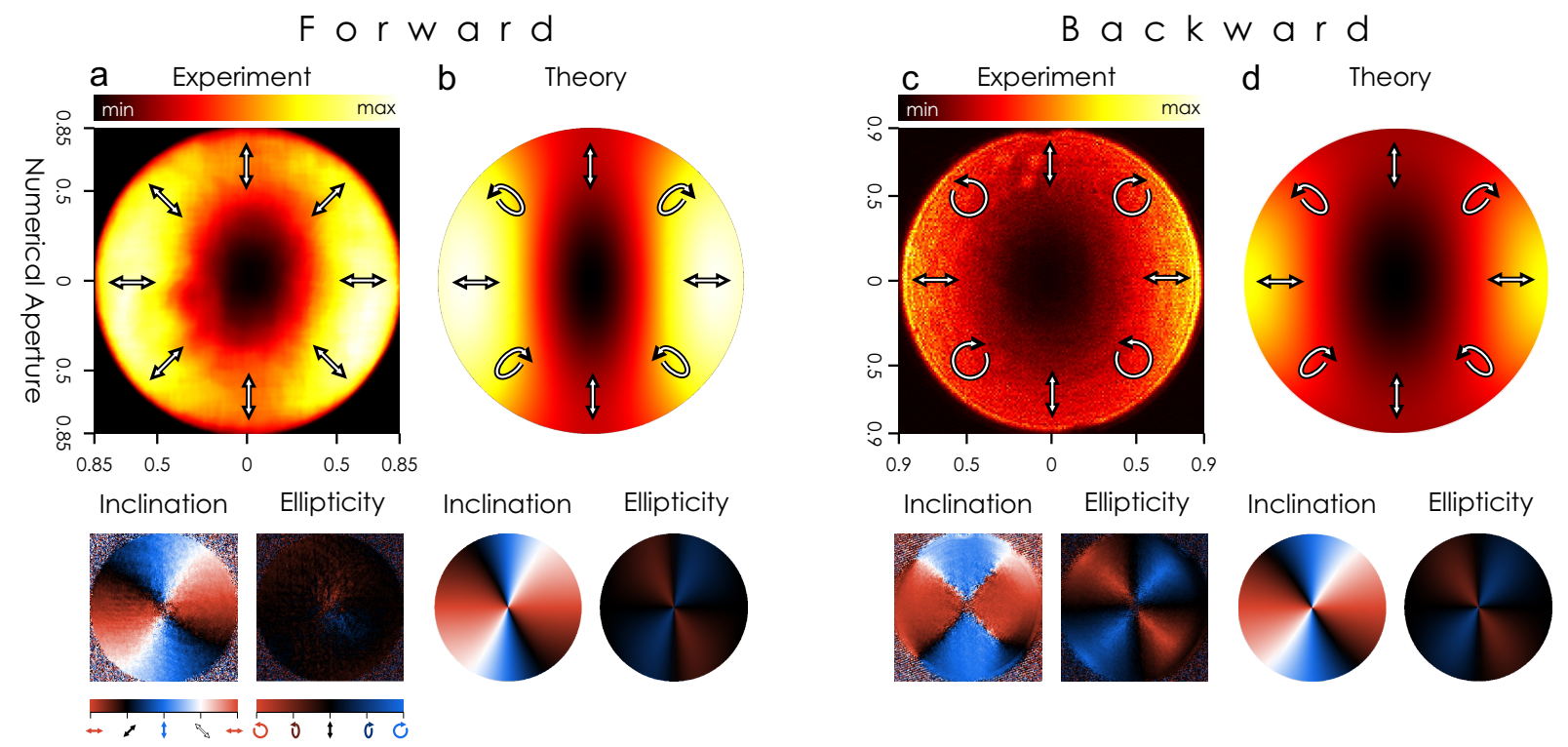

Figure 5: Directionality and polarization diagrams of the second harmonic. Top row: Directionality diagrams in $(\mathrm{a}, \mathrm{b})$ forward and $(\mathrm{c}, \mathrm{d})$ backward directions. (a,c) Experimental measurements, $(\mathrm{b}, \mathrm{d})$ theoretical calculations. Arrows visualize polarization states. Bottom row: Experimentally retrieved $(\mathrm{a}, \mathrm{b})$ and theoretically calculated (c,d) polarization inclination angles and ellipticity for the directionality cases of the top row. The incident beam polarization is vertical.

at the SH frequency, as shown with the arrows in Fig. 5(a). In particular, in experiment we observe nearly-perfect radial polarization of the $\mathrm{SH}$ in forward direction. In the backward direction, the polarization state is more complex with polarization inclination having radial structure and ellipticity ranging from nearly-circular to linear [Fig. 5(c)]. We also calculate the polarization of the SH beam numerically [see Figs. 5(b,d)]. While the numerical calculations predict similar polarization states in forward and backward directions, we observe some differences between theory and experiment. These can be attributed to the slight nonuniformity on the surface of AlGaAs as the $\mathrm{BCB}$ does not fully cover the nanodisks as well as due to possible imperfections in nanofabrication.

The nonlinear generation of the vector beams from our AlGaAs nanoantennas can be intuitively understood by the excitation of Mie-type multipoles at the $\mathrm{SH}$ frequency. In the simplest exemplary case, the vector beam of radial polarization can be emitted by an electric dipole oriented along the optical axis of the disk antenna. Indeed in our case, higher- 
order multipoles are excited at the $\mathrm{SH}$ wavelength. The superposition of these multipolar contributions is what governs the output polarisation state, which can be highly nontrivial and can be engineered for a specific application. Indeed, while in forward direction we observe polarization close to radial polarization state, the backward polarization state is of a more general nature.

In summary, we have studied the radiation pattern and polarization state of the SH emission from AlGaAs nanodisk antennas. We have shown that nonlinear conversion efficiencies exceeding $10^{-3}$ can be achieved, so such antennas can be applied for functional nonlinear devices at the nanoscale. In particular, nonlinear nanoscale light sources of vector beams with designer polarisation state, e.g. radial polarization have been experimentally demonstrated, for the first time to our knowledge. Our results open new avenues for novel nonlinear imaging, ${ }^{39}$ as well as application as bright fluorescent markers for bio-imaging, as well as for design of efficient nonlinear holograms. ${ }^{40}$

\section{Methods.}

Sample fabrication: We used metal-organic chemical vapour deposition (MOCVD) to grow $20 \mathrm{~nm}$ AlAs and $300 \mathrm{~nm}$ AlGaAs (20\% Al fraction) on a GaAs wafer. Patterned $\mathrm{SiO}_{2}$ masks were fabricated on the $\mathrm{AlGaAs}$ layer, using conventional e-beam lithography procedure. $\mathrm{SiO}_{2}$ layer was deposited via Plasma-enhanced chemical vapor deposition (PECVD). Then $\mathrm{SiO}_{2}$ disks were transferred to AlGaAs, AlAs and GaAs by reactive ion etching (RIE) technique. Subsequently, surface treatment has taken place via $\mathrm{Cl}_{2}$ purging by inductively coupled plasma (ICP) machine, in order to decrease the adhesion between BCB polymer and GaAs main wafer. $\mathrm{SiO}_{2}$ masks and $\mathrm{AlAs}$ layers were removed by hydrofluoric acid, which resulted in having AlGaAs disks sitting on GaAs wafer with minimum adhesion. The following step was the spin coating of $4 \mu \mathrm{m}$ BCB layer on the sample, then curing and bonding it to a thin glass substrate [see Fig. 1(c)]. Finally, the glass substrate with the BCB layer on top, 
containing AlGaAs disks, was peeled-off from the main GaAs wafer.

Optical characterization: The transmission through the AlGaAs nanodisk arrays of size $40 \times 40 \mu \mathrm{m}$ and periodicity of $1 \mu \mathrm{m}$ was measured using a home-built optical transmission system with a white-light source (tungsten halogen light bulb) and a spectrometer (Princeton Instruments Acton SP 2300 monochromator with Andor DU490A-1.7 InGaAs array detector). The scattering cross-sections were calculated as $\ln (1-T)$, where $T$ is the measured transmission, normalised to substrate.

For the SHG experiments, we place a single nanodisk in a focal spot of two confocal air objective lenses: Olympus LCPlanNIR (0.85 NA, 100× infrared) for focusing of the FW and Olympus MPlanFLN (0.9 NA, 100× visible) for collection of the SH. We measure a diameter

of the focused pump laser beam by performing knife-edge experiments, and ensure that the pump beam is close to a diffraction limit of $2.2 \mu \mathrm{m}$. The substrate side faces the visible objective. Thus, the objective lens Olympus MPlanFLN collects the SH from an individual disk in Forward direction, and the lens Olympus LCPlanNIR collects the SH radiation in Backward direction. The pump laser is a pulsed $\mathrm{Er}^{3+}$-doped fiber laser $(\sim 500 \mathrm{fs}$, repetition rate of $5 \mathrm{MHz}$ ) operating at a wavelength of $1556 \mathrm{~nm}$. At the laser output we employ a quarter-wave plate and a half-wave plate to control the output polarization. We use two cooled CCD cameras to detect the SH radiation. In forward direction a notch filter blocks the pump laser. In backward direction a dichroic mirror is used in front of the objective lens to direct the backward SH onto the camera.

\section{Acknowledgements}

The authors acknowledge the support by the Australian Research Council and participation in the Erasmus Mundus NANOPHI project, contract number 2013 5659/002-001. We thank Igal Brener, Giuseppe Leo, and Isabelle Staude for the useful discussions. The authors acknowledge the use of the Australian National Fabrication Facility (ANFF), the ACT Node. 


\section{References}

(1) Novotny, L.; van Hulst, N. Nat Photon 2011, 5, 83-90.

(2) Fu, Y. H.; Kuznetsov, A. I.; Miroshnichenko, A. E.; Yu, Y. F.; Luk'yanchuk, B. Nat. Commun. 2013, 4, 1527.

(3) Person, S.; Jain, M.; Lapin, Z.; Senz, J. J.; Wicks, G.; Novotny, L. Nano Lett. 2013, 13, 1806-1809.

(4) Staude, I.; Miroshnichenko, A. E.; Decker, M.; Fofang, N. T.; Liu, S.; Gonzales, E.; Dominguez, J.; Luk, T. S.; Neshev, D. N.; Brener, I.; Kivshar, Y. ACS Nano 2013, 7, $7824-7832$.

(5) Giannini, V.; Fernndez-Domnguez, A. I.; Heck, S. C.; Maier, S. A. Chem. Rev. 2011, 111, 3888-3912.

(6) Curto, A. G.; Volpe, G.; Taminiau, T. H.; Kreuzer, M. P.; Quidant, R.; van Hulst, N. F. Science 2010, 329, 930-933.

(7) Kruk, S. S.; Decker, M.; Staude, I.; Schlecht, S.; Greppmair, M.; Neshev, D. N.; Kivshar, Y. S. ACS Photon. 2014, 1, 1218-1223.

(8) Eizner, E.; Avayu, O.; Ditcovski, R.; Ellenbogen, T. Nano Lett. 2015, 15, 6215-6221.

(9) Palomba, S.; Novotny, L. Nano Lett. 2009, 9, 3801-3804.

(10) Zhang, Y.; Wen, F.; Zhen, Y.-R.; Nordlander, P.; Halas, N. J. Proc. Nat. Acad. Sci. 2013, 110, 9215-9219.

(11) Zhang, Y.; Zhen, Y.-R.; Neumann, O.; Day, J. K.; Nordlander, P.; Halas, N. J. Nat. Commun. 2014, 5, 4424.

(12) Lippitz, M.; van Dijk, M. A.; Orrit, M. Nano Lett. 2005, 5, 799-802. 
(13) Zhang, Y.; Grady, N. K.; Ayala-Orozco, C.; Halas, N. J. Nano Lett 2011, 11, 55195523.

(14) Wolf, D.; Schumacher, T.; Lippitz, M. Nat. Commun. 2016, 7, 10361.

(15) Gennaro, S. D.; Rahmani, M.; Giannini, V.; Aouani, H.; Sidiropoulos, T. P. H.; NavarroCa, M.; Maier, S. A.; Oulton, R. F. Nano Lett. 2016, 10.1021/acs.nanolett.6b02485.

(16) Aouani, H.; Navarro-Cia, M.; Rahmani, M.; Sidiropoulos, T. P. H.; Hong, M.; Oulton, R. F.; Maier, S. A. Nano Lett. 2012, 12, 4997-5002.

(17) Thyagarajan, K.; Rivier, S.; Lovera, A.; Martin, O. J. Opt. Express 2012, 20, 1286012865 .

(18) Czaplicki, R.; Husu, H.; Siikanen, R.; Mäkitalo, J.; Kauranen, M.; Laukkanen, J.; Lehtolahti, J.; Kuittinen, M. Phys. Rev. Lett. 2013, 110, 093902.

(19) Celebrano, M.; Wu, X.; Baselli, M.; Grossmann, S.; Biagioni, P.; Locatelli, A.; De Angelis, C.; Cerullo, G.; Osellame, R.; Hecht, B.; Duó, L.; Ciccacci, F.; Finazzi, M. Nat. Nanotechn. 2015, 10, 412-417.

(20) Metzger, B.; Gui, L.; Fuchs, J.; Floess, D.; Hentschel, M.; Giessen, H. Nano Lett. 2015, 15, 3917-3922.

(21) Grinblat, G.; Rahmani, M.; Cortés, E.; Caldarola, M.; Comedi, D.; Maier, S. A.; Bragas, A. V. Nano Lett. 2014, 14, 6660-6665.

(22) Kuznetsov, A. I.; Miroshnichenko, A. E.; Brongersma, M. L.; Kivshar, Y. S.; Lukyanchuk, B. Science 2016, in press.

(23) Shcherbakov, M. R.; Neshev, D. N.; Hopkins, B.; Shorokhov, A. S.; Staude, I.; MelikGaykazyan, E. V.; Decker, M.; Ezhov, A. A.; Miroshnichenko, A. E.; Brener, I.; Fedyanin, A. A.; Kivshar, Y. S. Nano Lett. 2014, 14, 6488-6492. 
(24) Carletti, L.; Locatelli, A.; Stepanenko, O.; Leo, G.; Angelis, C. D. Opt. Express 2015, 23, 26544-26550.

(25) Kauranen, M.; Zayats, A. V. Nat. Photon. 2012, 6, 737-748.

(26) Smirnova, D. A.; Khanikaev, A. B.; Smirnov, L. A.; Kivshar, Y. S. ACS Photon. 2016, 10.1021/acsphotonics.6b00036.

(27) Grinblat, G.; Li, Y.; Nielsen, M. P.; Oulton, R. F.; Maier, S. A. Nano Lett. 2016, 16, $4635-4640$.

(28) Yang, Y.; Wang, W.; Boulesbaa, A.; Kravchenko, I. I.; Briggs, D. P.; Puretzky, A.; Geohegan, D.; Valentine, J. Nano Lett. 2015, 15, 7388-7393.

(29) Chen, R.; Crankshaw, S.; Tran, T.; Chuang, L. C.; Moewe, M.; Chang-Hasnain, C. Appl. Phys. Lett. 2010, 96.

(30) Grange, R.; Brnstrup, G.; Kiometzis, M.; Sergeyev, A.; Richter, J.; Leiterer, C.; Fritzsche, W.; Gutsche, C.; Lysov, A.; Prost, W.; Tegude, F.-J.; Pertsch, T.; Tnnermann, A.; Christiansen, S. Nano Lett. 2012, 12, 5412-5417.

(31) Bautista, G.; Mkitalo, J.; Chen, Y.; Dhaka, V.; Grasso, M.; Karvonen, L.; Jiang, H.; Huttunen, M. J.; Huhtio, T.; Lipsanen, H.; Kauranen, M. Nano Lett. 2015, 15, 15641569.

(32) Fan, W.; Zhang, S.; Malloy, K. J.; Brueck, S. R. J.; Panoiu, N. C.; Osgood, R. M. Opt. Express 2006, 14, 9570-9575.

(33) Pu, M.; Ottaviano, L.; Semenova, E.; Yvind, K. Optica 2016, 3, 823-826.

(34) Carletti, L.; Locatelli, A.; Neshev, D.; Angelis, C. D. ACS Photon. 2016, 10.1021/acsphotonics.6b00050. 
(35) Gili, V. F.; Carletti, L.; Locatelli, A.; Rocco, D.; Finazzi, M.; Ghirardini, L.; Favero, I.; Gomez, C.; Lemaître, A.; Celebrano, M.; Angelis, C. D.; Leo, G. Opt. Express 2016, 24, 15965-15971.

(36) Liu, S.; Keeler, G. A.; yuanmu Yang,; Reno, J. L.; Sinclair, M. B.; Brener, I. Efficient second harmonic generation from GaAs all-dielectric metasurfaces. Conference on Lasers and Electro-Optics. 2016; p FM2D.6.

(37) Zhan, Q. Adv. Opt. Photon. 2009, 1, 1-57.

(38) Hugonin, J. P.; Lalanne, P. Reticolo software for grating analysis, Institut d'Optique, Orsay, France (2005).

(39) de Aguiar, H. B.; Gigan, S.; Brasselet, S. ArXiv e-prints 2016, arXiv:1603.07092.

(40) Almeida, E.; Bitton, O.; Prior, Y. ArXiv e-prints 2015, arXiv:1512.07899. 
Graphical TOC Entry

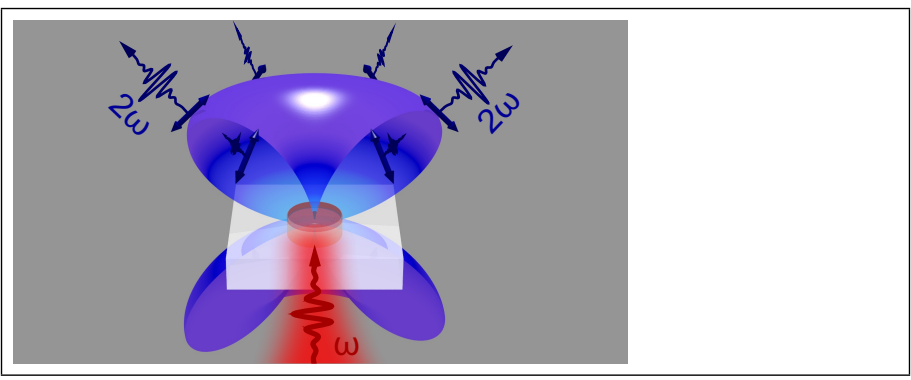

